\title{
Mechanism of SEMA3B gene silencing and clinical significance in glioma
}

\author{
C.H. Pang, W. Du, J. Long and L.J. Song \\ Department of Neurosurgery, The First Affiliated Hospital, Zhengzhou University, \\ Zhengzhou, China \\ Corresponding author: L.J. Song \\ E-mail: songlaijun99@163.com \\ Genet. Mol. Res. 15 (1): gmr.15017664 \\ Received September 17, 2015 \\ Accepted January 4, 2016 \\ Published March 18, 2016 \\ DOI http://dx.doi.org/10.4238/gmr.15017664
}

ABSTRACT. The aim of the current study was to explore mechanisms of SEMA3B gene expression and its clinical significance in glioma, and provide a theoretical foundation for investigating individualized treatment in glioma. Paraffin-embedded tissues from 43 patients with a confirmed clinical diagnosis of glioma following neurosurgery at the First Affiliated Hospital of Zhengzhou University from December 2013 to April 2014 were selected randomly. An additional three normal brain tissues were obtained following encephalic decompression excision due to acute craniocerebral injury in the same period, which were used as the control group. Immunohistochemical staining for vascular endothelial growth factor was performed on the glioma tissues from the 43 patients. Genomic DNA was extracted for bisulfate conversion and sequencing. SEMA3B was fully expressed in the three normal brain tissues, and incompletely expressed in the 43 glioma tissues, with a lack of expression in $48.8 \%(21 / 43)$ of samples. Moreover, $58 \%$ of high-grade gliomas (grade III and IV) lacked SEMA3B expression, which was significantly more than those that lacked expression (20\%) in low-grade gliomas (grade I and II), indicating that, as the clinical pathological grade increased, SEMA3B expression decreased. The occurrence and development of malignant tumors is a product of multiple genes and other factors. Here, we provide theoretical basis for 
glioma development and prognosis involving DNA-methylation driven silencing of SEMA3B, and thus, SEMA3B is a potential target for directed treatments against glioma.

Key words: SEMA3B; Glioma; Immunohistochemistry; DNA methylation

\section{INTRODUCTION}

Tumor occurrence and development is a process involving multiple genes and other factors, and has multiple stages. Malignant cancer can be considered a genomic disease, mainly characterized by the activation of proto-oncogenes (growth factors and their receptors, signal transduction factors, and gene transcriptional regulatory factors, etc.) and the inactivation of tumor suppressor genes. DNA methylation is an important molecular mechanism in the inactivation of tumor suppressor genes, and occurs in the promoter region of target genes (Karayan-Tapon et al., 2008). The overproliferation of glioma cells accelerates the progression and metastasis of the cancer, thereby causing further gene mutations, inducing excessive or abnormal gene expression, and inducing the inactivation of apoptosis signals within the tumor cells. In addition to these aberrant changes, the apoptosis of excessively proliferating cells, cell repair mechanisms, and the inhibition of tumor growth are induced.

The SEMA3B gene is now considered the 19th candidate tumor suppressor gene (TSG) (Nair et al., 2007). SEMA3B is involved in signal transduction pathways and its biological functions are associated with the combination of some receptors, which plays an important role in the formation of new blood vessels, in tumorigenesis, and in immune responses. In the process of its tumor suppression function, SEMA3B interacts with associated proteins to ultimately form a signal transduction pathway, which helps to reduce the occurrence of glioma, and thus this pathway may provide new targets for the treatment of glioma.

Glioblastoma multiforme (GBM) has been found to be the most common and most aggressive primary malignant brain tumor, and affects 25,000 patients yearly (Deorah et al., 2006). Currently, treatments for this disease include surgical resection and radiation therapy (RT) in combination with postoperative medication. However, the survival rate of GBM patients is still low. It is reported that the overall survival of GBM is 14.6 months and that the two-year postoperative survival rate is $26.5 \%$. The proliferation and diffuse infiltration of microvessels are two biological characteristics of glioma cells, which are also related to difficulties in curing this disease. Additionally, the tumor cells infiltrate normal brain tissue; therefore, it is challenging to remove all tumor cells during surgery. Presently, there are many specific treatments for these fatal tumor cells (Cohen et al., 1993). Furthermore, patients who cannot undergo surgery or bear postoperative recurrence and/or metastasis may consider drug therapies that can control tumor growth to a certain extent. Therefore, it is necessary to develop new drugs that can specifically target tumor cells, have low toxicity towards healthy cells, are safe, and exhibit strong antitumor effects for the treatment and prevention of recurrence of GBM.

\section{MATERIAL AND METHODS}

\section{Patients and tissue samples}

Paraffin-embedded blocks of glioma tissues clinically diagnosed after surgical removal were randomly selected from the Neurosurgery Department at the First Affiliated Hospital of 
Zhengzhou University from December 2013 to April 2014. Selected tissues were from patients that had no preoperative tumor treatment, and the postoperative pathological diagnosis for each tissue was glioma, of which there were 4, 25, 20, and 20 cases of grade-I, -II, -III, and -IV gliomas, respectively. Thirty-six cases were male, 33 cases were female. The ratio of males to females was 1.07:1, and the patient ages ranged from 17 to 70 years (mean $48.37 \pm 12.06$ years). All tissue blocks were cut into $5-\mu \mathrm{m}$ sections by professional personnel. Additionally, three normal brain tissues removed by intracranial pressure due to acute traumatic brain injury were selected as the control group. Tissues from the control group were cryopreserved using liquid nitrogen and stored until subsequent use. Written informed consent was obtained for all study participants.

\section{Reagents and instruments}

The following reagents and instruments were used in the study: Ultra Sensitive TMSHypersensitive Kit (Maixin Biotechnology Company, Fuzhou, China); DAB Coloration Kit (Maixin Biotechnology Development Company, Fuzhou, China); Plasmid Extraction Kit (Sangon Biological Engineering Co., Ltd., Shanghai, China); SK8161; sappanwood blank cell-staining fluid (Maixin Biotechnology Development Company, Fuzhou, China); CpG Assay (200) (Qiagen, Germany); DNA Extraction Kit (Qiagen, Germany); $\mathrm{NaHSO}_{3}$ (analytically pure, Sangon); Taq DNA polymerase SC0010 and dNTPs (Sangon); hydroquinone (Sangon); 3730 Sequencing Analysis Meter (Applied Biosystems, Carlsbad, CA, USA); SW-CJ-1D Clean Bench (Sue Clean Equipment Factory, Jiangsu); DK-8D Electric Heating Thermostatic Water Bath (Senxin Experimental Instrument Co., Ltd., Shanghai, China); YXJ-2 Centrifuge (Xiangyi Centrifugal Machine Instrument Co., Ltd.); Liquid-Moving Machine (scope 100-1000, 20-200, 0.5-10 mL) (BBI Life Sciences, Shanghai, China).

\section{Immunohistochemical staining of vascular endothelial growth factor (VEGF) in glioma tissue}

The Ultra Sensitive TMS-Hypersensitive Kit was used to stain sliders as per the instructions of the manufacturer. The DAB reagent was prepared simultaneously with the last of these three PBS washes; the PBS was then removed, and $100 \mu \mathrm{L}$ fresh DAB liquid was added to each section. Next, the presence of yellow staining was observed after 3-10 min under microscopy.

\section{Genomic DNA extraction from the paraffin embedded samples}

Genomic DNA was extracted from the paraffin-embedded sample blocks following manufacture instructions (note: Buffer AL and methyl alcohol were mixed in advanced).

\section{DNA bisulfate conversion}

The DNA was first melted for use in the bisulfate reaction. Next, $800 \mu \mathrm{L}$ enzyme-free water was added to dissolve each uniform mixture of hydrogen sulfite. The solutions were mixed for 5 min until the full dissolution of sodium hydrogen sulfite, and then the bisulfate reaction mixtures were prepared in 200- $\mu \mathrm{L}$ PCR tubes according to the volumes shown in Table 1. 
Table 1. Bisulfate reaction mixtures.

\begin{tabular}{l|c}
\hline Component & Volume per reaction $(\mu \mathrm{L})$ \\
\hline DNA solution $(1-2 \mu \mathrm{g})$ & 10 \\
\hline Rnase-free water & 10 \\
\hline Bisulfite mix (dissolved) & 85 \\
\hline DNA protect buffer & 35 \\
\hline Total volume & 140 \\
\hline
\end{tabular}

The PCR tubes were covered; the bisulfate reaction mixtures were mixed vigorously; the reaction mixtures were incubated at room temperature; and then the DNA bisulfate conversions were carried out in a thermal cycler with conditions shown in Table 2.

Table 2. Thermal cycle conditions for bisulfate conversion.

\begin{tabular}{l|c|c}
\hline Step & Time $(\mathrm{min})$ & Temperature $\left({ }^{\circ} \mathrm{C}\right)$ \\
\hline Denaturation & 5 & 95 \\
\hline Incubation & 25 & 60 \\
\hline Denaturation & 5 & 95 \\
\hline Incubation & 85 & 60 \\
\hline Denaturation & 5 & 95 \\
\hline Incubation & 175 & 60 \\
\hline Store & Overnight & 20 \\
\hline
\end{tabular}

After thermal cycling and completion of the bisulfate conversions, the reaction mixtures were moved into 1.5-mL centrifuge tubes; $310 \mu \mathrm{L}$ fresh Buffer BL that included $10 \mu \mathrm{g} / \mathrm{mL}$ carrier RNA was added into each sample. The tubes were mixed and centrifuged briefly, $250 \mu \mathrm{L}$ 96-100\% ethyl alcohol was added to each sample and mixed for $15 \mathrm{~s}$ before centrifuging briefly to remove droplets in the tubes. EpiTect spin columns were placed into collection tubes; the complete bisulfite reaction mixtures were transferred to the EpiTect spin columns; the spin column/collection tubes were centrifuged at the maximum speed; the flow through was removed; and then the spin columns were put back into the collection tubes. Next, $500 \mu \mathrm{L}$ Buffer BW was added to each spin column; centrifuged again at maximum speed for $1 \mathrm{~min}$; the flow through was removed; and the columns were put back into the collection tubes. Then, $500 \mu \mathrm{L}$ Buffer BD was added to each spin column; the columns were incubated for $15 \mathrm{~min}$ at room temperature; centrifuged at maximum speed for 1 min; the flow through was removed; and the columns were put back into the collection tubes. Next, $500 \mu \mathrm{L}$ Buffer BW was added to each spin column; the columns were centrifuged at the maximum speed for $1 \mathrm{~min}$; this last step was repeated; the spin columns were placed into 2-mL collection tubes; centrifuged at the maximum speed for $1 \mathrm{~min}$; and any residual liquid was removed. The spin columns (cap opened) were put into new 1.5-mL clear centrifuge tubes; heated at $56^{\circ} \mathrm{C}$ for $5 \mathrm{~min}$; put into clear centrifuge tubes; $20 \mu \mathrm{L}$ Buffer EB was added to the center of each membrane; and then finally the columns were centrifuged at $12,000 \mathrm{rpm}(15,000 \mathrm{~g})$ for $1 \mathrm{~min}$ to elute the purified DNA.

\section{Gene sequencing}

Positive bands were cut off for recycling DNA as per the instructions of the manufacturer, and then gene was sequenced under the following conditions (Table 3). 


\section{Table 3. Reaction system.}

\begin{tabular}{l|c|r}
\hline Degeneration & 25 -time cycles & Constant temperature \\
\hline $96^{\circ} \mathrm{C}$ for $1 \mathrm{~min}$ & $96^{\circ} \mathrm{C}$ for $10 \mathrm{~s}: 50^{\circ} \mathrm{C}$ for $5 \mathrm{~s}: 60^{\circ} \mathrm{C}$ for 4 min & $4^{\circ} \mathrm{C}$ \\
\hline
\end{tabular}

Finally, the products were purified and detected.

\section{Statistical method}

The GraphPad Prism 5 Software (San Diego, CA, USA) was used for one-way analysis of variance (ANOVA) and correlation analyses, and data are reported as means \pm SD. Results were considered to be statistically significant at $\mathrm{P}<0.05$.

\section{RESULTS}

\section{Methylation of the SEMA3B gene promoter in glioma}

Studies have shown that methylation in the promoter regions of TSGs is the most common cause of their inactivation, which indicates that the most direct way to analyze mechanisms of TSG silencing in glioma is to analyze genetics and epigenetics. Thus, this research may allow us to better understand and explain glioma development. As mentioned, the most direct epigenetic mechanism for TSG silencing is DNA methylation, and one such TSG that can be affected by this is SEMA3B. Therefore, we analyzed the CpG methylation status of the SEMA3B gene in glioma tissues using the $\mathrm{CpG}$ methylation pyrophosphate test of the 3p21.3 area (Figure 1), and conducted a quantitative analysis for the degree of methylation at the target locus through detecting the rate of $\mathrm{C} / \mathrm{T}$ at the $\mathrm{CpGs}$ at that locus. Our results revealed that the SEMA3B gene may be a potential methylation locus in glioma.

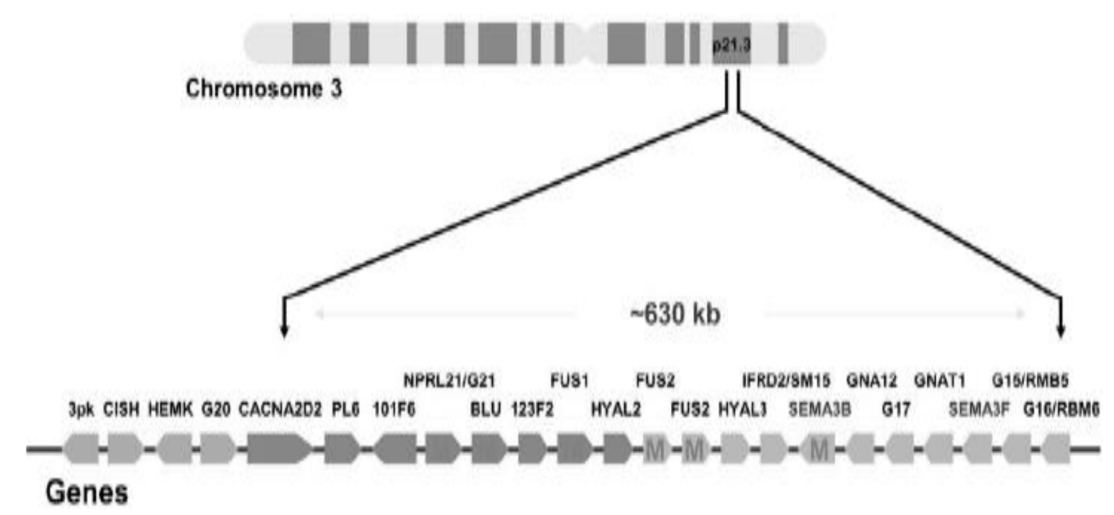

Figure 1. Chromosomal location of the SEMA3B gene.

Through the detection of methylation in the SEMA3B promoter region in tissues from 43 glioma cases and from 3 normal brain tissues (Table 4), we found that the three normal brain tissues were unmethylated and that 21 glioma cases were methylated with a methylation rate of $48.8 \%$ (21/43). Moreover, we analyzed the specific cites of methylation using PCR and pyrosequencing methods, and found five methylated loci (Figure 2). 
Table 4. Clinical information of glioma patients from whom tissue samples were used for the methylation analysis.

\begin{tabular}{|c|c|c|c|c|c|c|}
\hline ID & Gender & Age & PD & Grade & Methylated & Unmethylated \\
\hline 1 & $\mathrm{~F}$ & 28 & NB & - & - & - \\
\hline 2 & M & 30 & NB & - & - & - \\
\hline 3 & M & 45 & NB & - & - & - \\
\hline 4 & $\mathrm{~F}$ & 30 & PA & I & - & - \\
\hline 5 & $\bar{F}$ & 17 & PA & $T$ & - & - \\
\hline 6 & $M$ & 48 & MOA & II & + & - \\
\hline 7 & M & 69 & ODG & II & - & - \\
\hline 8 & M & 65 & LGA & II & - & - \\
\hline 9 & $\mathrm{~F}$ & 53 & MOA & II & - & - \\
\hline 10 & $\mathrm{~F}$ & 53 & LGA & II & - & - \\
\hline 11 & $\mathrm{~F}$ & 43 & MOA & III & + & - \\
\hline 12 & $\mathrm{~F}$ & 55 & AA & III & + & - \\
\hline 13 & $\mathrm{~F}$ & 65 & AMOA & III & + & - \\
\hline 14 & M & 50 & $\mathrm{AO}$ & III & + & - \\
\hline 15 & $\mathrm{~F}$ & 54 & LGA & IIII & - & - \\
\hline 16 & M & 61 & GBM & IV & + & - \\
\hline
\end{tabular}

$\mathrm{NB}=$ normal brain tissue; $\mathrm{PD}=$ pathological diagnosis; $\mathrm{PA}=$ hair-cell type astrocytoma; $\mathrm{LGA}=$ dispersivity astrocytoma; $\mathrm{ODG}=$ less glioma; $\mathrm{MOA}=$ oligodendroglioma; $\mathrm{AMOA}=$ variant and hybrid oligodendroglioma; $\mathrm{AA}=$ variant astrocytoma; $\mathrm{AO}$ = variant oligodendroglioma; $\mathrm{GBM}=$ colloid mother tumor; (+) positive; (-) negative.

1

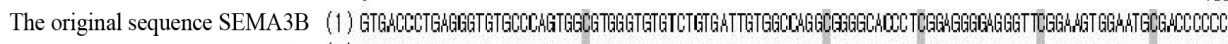

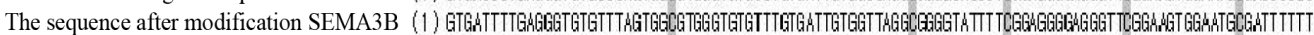

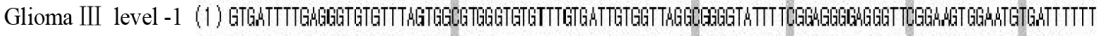

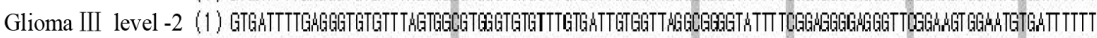

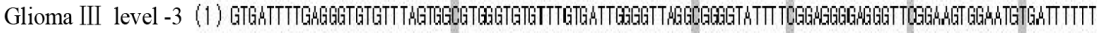

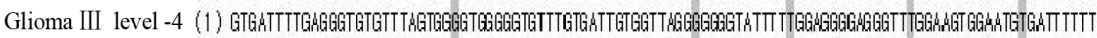

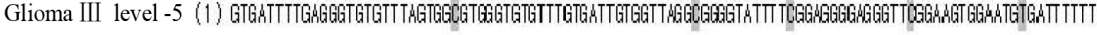

1

100

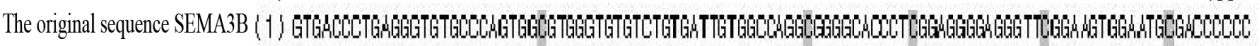

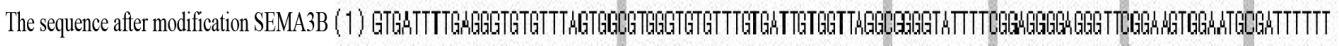

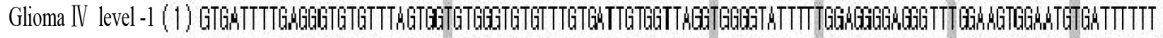

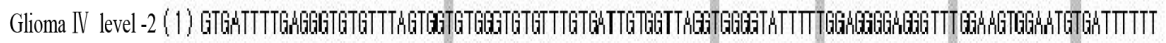

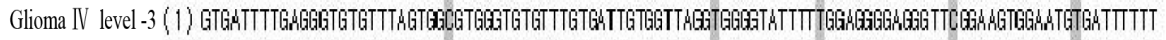

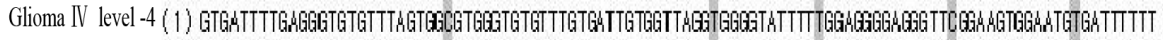

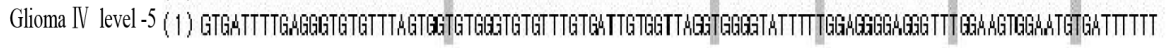

1

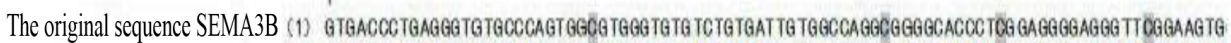

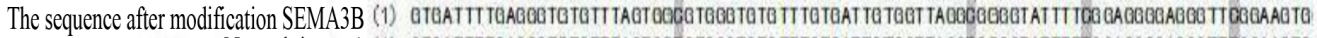

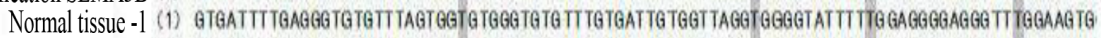

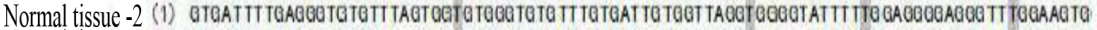

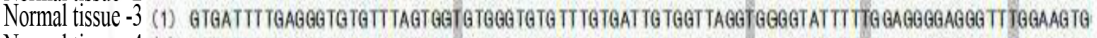
Normal tissue -4 (1) GTGATTTTGACCCTGTOTTTAGTCGTGTGCGTCTO TTTGTGATTGTGGTTACGTGGGGTATTTTTG CACGCGAGCG TTTCGAAGTO Normal tissue -5 (1) GTGATTTTGAGGGTGTTTTAGTGGTGTGGGTGTG TTTGTGATTGTGGTTAGGTGGGGTATTTTTGGAGGGGAGGGTTTGGAAGT

Figure 2. SEMA3B sequence alignment following bisulfite conversion of representative grade-III and -IV glioma tissues and normal brain tissues. 


\section{Methylation status of SEMA3B and the clinical pathological characteristics of glioma patients}

We found that methylation in the promoter region was not detected in grade-I glioma tissues, while the methylation rates of grade-II, -III, and -IV gliomas were 25\% (2/8), 67\% (12/18), $47 \%$ (7/15), respectively, and the data are shown in Table 4. Through the comparison of methylation rates in gliomas of high and low grades, we discovered that 58\% (19/33) of SEMA3B promoters in the high-grade glioma group were methylated, whereas only $20 \%(2 / 10)$ were methylated in the low-grade glioma group. A general increasing trend in the methylation of the SEMA3B gene was observed with increasing pathological grade of the samples, which also indirectly suggests that SEMA3B gene silencing and methylation in the promoter region are correlated. Statistical analysis revealed that the degree of methylation in normal brain tissue and low-grade glioma tissues was significantly different from that in high-grade glioma tissues (Table 5). Therefore, we can consider methylation in the promoter region of the SEMA3B gene as one of the mechanisms of its downregulation in glioma.

Table 5. Clinical pathological characteristics of 3 normal brain tissues and tissues from 43 cases of glioma and SEMA3B methylation status from these tissues.

\begin{tabular}{l|l|c|c|c|c}
\hline Characteristic & Types & Percentage (\%) & Unmethylated (\%) & Methylated (\%) & Methylation rate (\%) \\
\hline \multirow{2}{*}{ Gender } & Male & $26(56.5)$ & $9(19.6)$ & $17(36.9)$ & 65.4 \\
\cline { 2 - 6 } & Female & $20(43.5)$ & $6(13.0)$ & $14(30.5)$ & 70.0 \\
\hline \multirow{2}{*}{ Age } & $\leq 60$ & $35(76.1)$ & $15(32.6)$ & $20(43.5)$ & 57.1 \\
\cline { 2 - 6 } & $>60$ & $11(23.9)$ & $2(4.3)$ & $9(19.6)$ & 81.8 \\
\hline \multirow{2}{*}{ Tissues } & Normal brain tissues & $3(6.5)$ & $0(0)$ & $3(100.0)$ & 100.0 \\
\cline { 2 - 6 } & I/II & $10(21.7)$ & $8(17.4)$ & $19(4.3)$ & 20.0 \\
\cline { 2 - 6 } & III/IV & $33(71.7)$ & $14(30.4)$ & $26(56.5)$ & 67.6 \\
\hline Total & Glioma & $43(93.5)$ & $17(37.0)$ & & 60.5 \\
\hline
\end{tabular}

\section{DISCUSSION}

The SEMA3B gene belongs to the semaphorin/collapsin family, and mainly exists in the form of a secreted protein or membrane protein. Alterations in the human 3p21.3 chromosome area are often implicated in high incidences of cancer. For example, loss of heterozygosity of this area is more frequent in cancers such as in lung cancer, breast cancer, ovarian cancer, and cancers of other organs. The SEMA3B gene usually shows higher expression levels in normal tissues than those found in tumors. However, SEMA3B expression has been shown to be higher in highly metastatic tumors such as in lung cancer, but lower in melanoma cells, bladder cancer cells, and prostate cancer cells. Moreover, the genome in glioblastoma cells has shown a wide range of low methylation in specific areas (Kozlowski et al., 2010). This methylation pattern increases genetic instability, silences TP53 and PTEN TSGs, and activates oncogenes. Methylation usually occurs in the promoter $\mathrm{CpG}$ island of a gene, and methylation in these regions is related to expression of TSGs (McDonnell et al., 2001), such as those involved in DNA repair (Quiros et al., 2011), cell cycle (Rickles et al., 2001) and cell apoptosis (Saitoh et al., 1995; Liang et al., 2013), invasion (Suchorska et al., 2011), and migration.

Research has shown that SEMA3B gene expression is absent or reduced by more than $80 \%$ in lung cancer cell lines, and has homozygous deficiency in tissues from lung cancer. It was previously found that methylation in the promoter region of SEMA3B can reach $92 \%$ in gallbladder carcinoma, which indicated that high methylation of the SEMA3B gene participates in gallbladder 
carcinoma development (Jarjour et al., 2011). Expression of SEMA3B has been shown to increase cell apoptosis and inhibit the growth of human lung cancer cells, breast cancer and ovarian cancer cells, and of the A9 sarcoma cell line in mice. These findings strongly implicate SEMA3B in the inhibition of tumor cell growth. Additionally, SEMA3B plays a role in the process of mediated neural axon growth. Recent research on a variety of tumor cells has shown that the 5'-CpG island in the promoter of SEMA3B is highly methylated, which leads to low or absent expression of the gene in tumor cell lines. Notably, in small-cell lung cancer and nonsmall cell carcinoma, the deficient expression of the SEMA3B gene can predict the degree of malignancy and aggressiveness of tumors. In vitro transfection of the SEMA3B gene can reduce the proliferation and invasion ability of tumor cells, and can ablate the tumorigenicity of cancer cells in nude mice. Moreover, SEMA3B expression can inhibit the growth of malignant cells, which may explain the close relationship between SEMA3B gene expression and the occurrence of cancer.

In the current study, we detected the expression of SEMA3B by adopting RT-PCR methods. We found that in the three normal brain tissues, SEMA3B was fully expressed, whereas in the 43 glioma tissue samples, the SEMA3B expression rate was $48.8 \%(21 / 43)$. Interestingly, with increases in clinical pathologic grade, we observed decreases in SEMA3B expression. Specifically, we found that the deficiency rate of SEMA3B expression in highgrade glioma (grades III and IV) was 58\%, which was significantly higher than the deficiency rate $(20 \%)$ in low-grade glioma (grades I and II), indicating that higher clinical pathological grades were associated with more significant decreases in SEMA3B gene expression. These differences in SEMA3B expression are likely to affect glioma prognosis, and may also participate in the development of these tumors. The physiological deficiency of the SEMA3B gene may have resulted in the loss of its expression, and this may directly cause the disease or aggravate the development of the illness, which further illustrates that SEMA3B plays an important role as a TSG in glioma. The normal physiological function of SEMA3B is reduced in gliomas, which enhances their degree of malignancy. We also demonstrated that the deficiency rate in low-grade gliomas was significantly lower than that in high-grade gliomas, which may indicate that the high-grade cases will progress with faster growth patterns, and may be more easily transformed in the direction of malignancy. We can thus predict that high expression of SEMA3B is associated with a positive prognosis in glioma patients.

In conclusion, DNA methylation continues to draw more and more attention in early diagnosis, prognostic evaluation, and targeted clinical treatments of different human cancers, and plays an important role in tumorigenesis and development. DNA methylation, the most common epigenetic event, does not change primary gene sequences, but rather can lead to changes in gene expression and to chromatin structure. Taken together, the results of the present study suggest that DNA methylation may be a potential target in gene-targeted therapies, and may be valuable in early diagnosis, prognostic evaluation, disease progress, and treatment of glioma.

\section{Conflicts of interest}

The authors declare no conflict of interest.

\section{ACKNOWLEDGMENTS}

I would like to express my sincere gratitude to all research workers engaged in this study. 


\section{REFERENCES}

Cohen P, Peehl DM, Stamey TA, Wilson KF, et al. (1993). Elevated levels of insulin-like growth factor-binding protein-2 in the serum of prostate cancer patients. J. Clin. Endocrinol. Metab. 76: 1031-1035.

Deorah S, Lynch CF, Sibenaller ZA and Ryken TC (2006). Trends in brain cancer incidence and survival in the United States: Surveillance, Epidemiology, and End Results Program, 1973 to 2001. Neurosurg. Focus 20: E1-E7. http://dx.doi. org/10.3171/foc.2006.20.4.E1

Jarjour AA, Durko M, Luk TL, Marçal N, et al. (2011). Autocrine netrin function inhibits glioma cell motility and promotes focal adhesion formation. PLoS One 6: e25408. http://dx.doi.org/10.1371/journal.pone.0025408

Karayan-Tapon L, Wager M, Guilhot J, Levillain P, et al. (2008). Semaphorin, neuropilin and VEGF expression in glial tumours: SEMA3G, a prognostic marker? Br. J. Cancer 99: 1153-1160. http://dx.doi.org/10.1038/sj.bjc.6604641

Kozlowski M, Kowalczuk O, Milewski R, Chyczewski L, et al. (2010). Serum vascular endothelial growth factors C and D in patients with oesophageal cancer. Eur. J. Cardiothorac. Surg. 38: 260-267. http://dx.doi.org/10.1016/j.ejcts.2010.01.061

Liang C, Guo S and Yang L (2013). All-trans retinoic acid upregulates VEGF expression in glioma cells in vitro. J. Biomed. Res. 27: 51-55. http://dx.doi.org/10.7555/JBR.27.20120048

McDonnell CO, Harmey JH, Bouchier-Hayes DJ and Walsh TN (2001). Effect of multimodality therapy on circulating vascular endothelial growth factor levels in patients with oesophageal cancer. Br. J. Surg. 88: 1105-1109. http://dx.doi.org/10.1046/ j.0007-1323.2001.01838.x

Nair PN, McArdle L, Cornell J, Cohn SL, et al. (2007). High-resolution analysis of 3p deletion in neuroblastoma and differential methylation of the SEMA3B tumor suppressor gene. Cancer Genet. Cytogenet. 174: 100-110. http://dx.doi.org/10.1016/j. cancergencyto.2006.11.017

Quiros S, Roos WP and Kaina B (2011). Rad51 and BRCA2--New molecular targets for sensitizing glioma cells to alkylating anticancer drugs. PLoS One 6: e27183. http://dx.doi.org/10.1371/journal.pone.0027183

Rickles FR, Shoji M and Abe K (2001). The role of the hemostatic system in tumor growth, metastasis, and angiogenesis: tissue factor is a bifunctional molecule capable of inducing both fibrin deposition and angiogenesis in cancer. Int. J. Hematol. 73: 145-150. http://dx.doi.org/10.1007/BF02981930

Saitoh Y, Kuratsu J, Takeshima H, Yamamoto S, et al. (1995). Expression of osteopontin in human glioma. Its correlation with the malignancy. Lab. Invest. 72: 55-63.

Suchorska B, Ruge M, Treuer H, Sturm V, et al. (2011). Stereotactic brachytherapy of low-grade cerebral glioma after tumor resection. Neuro-oncol. 13: 1133-1142. http://dx.doi.org/10.1093/neuonc/nor100 